\title{
Asesinato en el aire desde la mente del perpetrador: A bordo del Vuelo 9525 Germanwings
}

\author{
María ALDECUA-TEJERO, Citlalli Abigail GARCIA-MEZO, Martín INURRETA-DÍAZ, Leticia LIZAMA- \\ RUBIO, Carlos Daniel VELASCO-BONILLA, Nina Isabel MÉNDEZ-DOMÍNGUEZ
}

Escuela de Medicina. Universidad Marista de Mérida (México).

Autor para correspondencia: Nina Méndez-Dominguez. Correo electrónico: ninuxka@hotmail.com

Recibido el 27 de septiembre de 2018; aceptado el 10 de octubre de 2018.

Cómo citar este artículo: Aldecua Tejero M, Garcia Mezo CA, Inurreta Díaz M, Lizama Rubio L, Velasco Bonilla CD, Méndez Domínguez NI. Asesinato en el aire desde la mente del perpetrador: A bordo del Vuelo 9525 Germanwings. Rev Med Cine [Internet] 2019;15(3): 147-152.

DOI: http://dx.doi.org/10.14201/rmc2019153147152

\section{Resumen}

La salud mental es un componente fundamental del bienestar humano, cuando este componente se ve afectado en la vida de un individuo que tiene bajo su responsabilidad la vida de otras personas, nadie está exento de poder sufrir las consecuencias de dicha inestabilidad. Mayday Catástrofes Aéreas: Vuelo 9525 Germanwings - Asesinato en el aire (2016) relata lo acontecido en torno al copiloto responsable de la catástrofe, nos adentra en la mente del perpetrador, situándonos en el contexto en el cual se desenvolvía y permitiéndonos comprender en retrospectiva los puntos fundamentales que pudieron haber servido como señales de alarma y nos invita a reflexionar acerca de las vías de prevención que potencialmente pudieron haberse implementado. Si bien las condiciones orgánicas del copiloto mostraron ser saludables, una combinación de ansiedad y depresión detonadas por la carga laboral y la falta de pertenencia pudieron ser abordadas desde la medicina preventiva y el lifestyle medicine.

Los pilotos y copilotos componen un gremio de profesionistas expuestos a estilos de vida que pueden afectar su estabilidad y salud mental y es por ello que se resalta la importancia de la implementación de medidas preventivas, mismas que pudieron aplicarse para evitar la tragedia del Germanwings 9525 que despegó del Aeropuerto de Barcelona con ciento cincuenta personas a bordo, mas nunca llegó a su destino. En el presente artículo se discute la importancia de abordar mediante modelos multinivel la salud de los pilotos y copilotos a propósito del caso del Germanwings, desde el enfoque de la medicina preventiva.

Palabras clave: depresión; ansiedad; suicidio; medicina preventiva; lifestyle medicine.

\section{Murder in the air from the perpetrator's mind: On board Flight 9525 Germanwings}

\begin{abstract}
Summary
Mental health is a fundamental component of human well-being, but when this component is affected in the life of an individual who has the responsibility of other people's lives, no one is exempt from suffering the consequences of such instability. Mayday Air Catastrophes: Flight 9525 Germanwings - Murder in the Air (2016) recounts the events surrounding the co-pilot responsible for the catastrophe, while it takes us into the mind of the perpetrator, placing us in the context in which it developed and allowing us to understand in retrospect the fundamental points that could have served as alarm signals and invites us to reflect on the prevention routes that could potentially have been implemented. Although the organic conditions of the co-pilot showed to be healthy, a combination of anxiety and depression detonated by the workload and the lack of belonging could be addressed from preventive medicine and lifestyle medicine.

Pilots and co-pilots make up a guild of professionals exposed to lifestyles that can affect their stability and mental health and that is why the importance of the implementation of preventive measures is highlighted, which could be applied to avoid the tragedy of the Germanwings 9525 It took off from Barcelona Airport with a hundred and fifty passengers on board, but never reached its destination. In this article, we discuss the importance of addressing the health of pilots and co-pilots using the multilevel models in the Germanwings case, from the perspective of the preventive medicine.
\end{abstract}

Keywords: depression; anxiety; suicide; preventive medicine; lifestyle medicine. 


\section{Ficha técnica}

Título: Mayday Catástrofes Aéreas: Vuelo 9525 Germanwings - Asesinato en el aire.

Título original: MAYDAY.

Otros títulos: Mayday: catástrofes aéreas.

País/es: Canadá, EEUU, Reino Unido, Irlanda, Australia, África del Sur, España y Asia.

Año: 2016.

Director: Timothy Wolochatiuk.

Música: Anthony Rozankovic.

Fotografía: James Griffith, Mark Caswell.

Guión: Julia Nunes

Intérpretes: Noah Davis, Travis Jay Riley, Courtenay Stevens, Mark Mcgnnder, Jerome Bourgault, Elma Begovic, Monika Schurmann, Claire Macnabb, David Dawson, Peter Skrzypezak, Amanda Mitro.

Color: color.

Duración: 45 minutos.

Género: documental.

Sinopsis: El 24 de marzo de 2015 el vuelo 9525 de la aerolínea alemana Germanwings parte del aeropuerto Barcelona-El Prat en España con destino a Düsseldorf, Alemania; llevando a bordo 150 pasajeros donde al sobrevolar los Alpes franceses sufrirían un devastador accidente aéreo, que abriría una serie de investigaciones técnicas y criminales en las cuales se analizan los audios de la cabina así como la ruta que recorrió el avión para así llegar a un veredicto final, esclareciendo la razón de la catástrofe.

Productoras: Alex Bystram, Kim Bondi. Enlaces:

https://www.dailymotion.com/video/x6h9xx1

\section{Tráiler en español}

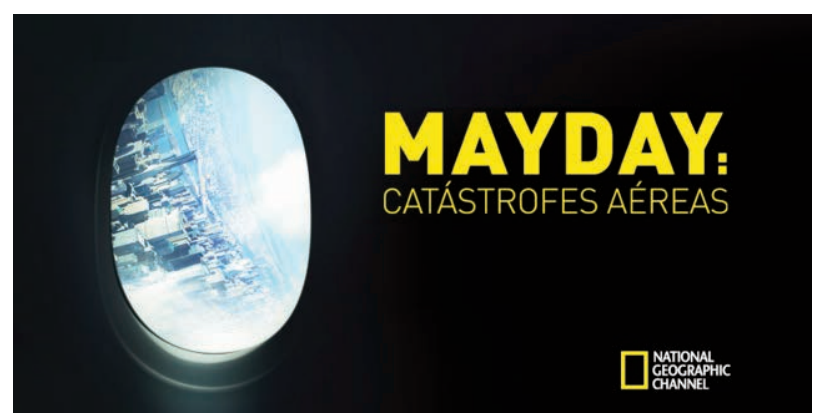

\section{Introducción}

La depresión mayor es un trastorno complejo, en la cual sus manifestaciones psicopatológicas se relacionan con la personalidad y dependen de la interacción entre factores genéticos y ambientales a lo largo del desarrollo ${ }^{1}$, según -Robert Bor psicólogo de la aviación describe en el documental Mayday Catástrofes Aéreas: Vuelo 9525 Germanwings - Asesinato en el aire "un problema de salud mental no tiene porque necesariamente traducirse en cambios de comportamiento".

No existe una sola causa aparente de este trastorno, suele ser una combinación que engloba factores genéticos, biológicos, psicológicos y ambientales ${ }^{2}$.

Las manifestaciones clínicas suelen presentarse durante un mínimo de dos semanas e interferir en actividades como el trabajo y las relaciones familiares, que incluyen: cambios de humor caracterizados por periodos de tristeza o irritabilidad, así como ciertos cambios psicofisiológicos, como lo son alteraciones en el patrón del sueño, el apetito y el deseo sexual; estreñimiento, pérdida de la capacidad de experimentar placer en actividades cotidianas, llanto, pensamientos suicidas y ralentización del habla y acciones ${ }^{3}$; baja autoestima y un aumento de la autocrítica y los niveles de perfeccionismo ${ }^{1}$.

En los individuos que han presentado un cuadro depresivo, tiende a incrementar el riesgo de morbilidad general, riesgo de conductas suicidas y como consecuencia, aumento de la mortalidad. De igual manera, existe la posibilidad de presentar más de un episodio durante un periodo de tiempo no determinado 4 .

Si los episodios depresivos son recurrentes o crónicos, hay un incremento del estrés en los familiares y amigos, también puede afectar la capacidad parental de la persona, teniendo esto como consecuencia disfunción ocupacional4.

Los trastornos depresivos no tratados incrementan el riesgo de suicidio o de diferentes actos violentos, aunado a esto, el abuso de algún tipo de droga, el desempleo y aislamiento social, aumentan las probabilidades del mismo 4 .

El consumo de algún tipo de droga, puede agravar la situación al grado de tener complicaciones físicas, y con esto poner en peligro tanto el bienestar del paciente, así como el de las personas que lo rodean. 
El copiloto Andreas Lübitz, no presentaba cambios de humor o de personalidad que pudiesen indicar que padecía depresión, esto trajo como consecuencia que las autoridades correspondientes no tuvieran el conocimiento de su condición, y lo dejarán pilotar el vuelo el 9525 de la aerolínea Germanwings, que terminaría como un accidente aéreo con un trasfondo mucho más trágico.

\section{Análisis desde el enfoque de la medicina preventiva}

Realizando un análisis desde un enfoque preventivo, se considera que el monitoreo constante del bienestar mental de los pilotos, así como la educación enfocada a la salud en la preparación de cualquier individuo sobre todo en las universidades de pilotaje es de gran importancia como prevención primaria, ya que al ser impartidos estos métodos se podría detectar a tiempo alguna enfermedad/trastorno como es la depresión y en otras circunstancias evitar el accidente aéreo. Controles psicométricos serían necesarios para poder detectar a tiempo alguna enfermedad mental.

Por otra parte, el impartir programas preventivos como pláticas acerca de la enfermedad y cómo detectarla sería una herramienta de gran utilidad para la sociedad, proporcionándoles la información necesaria, no sólo para percatarse por sí mismos, sino también para ser conscientes de la gravedad del problema y tener la oportunidad de brindarles ayuda profesional.

Andreas Lübitz se sometió a dichas pruebas psicológicas con diferentes médicos, obteniendo como resultado, cartas donde se sugería la suspensión laboral para no pilotar y ser ingresado en una institución psiquiátrica debido a su estado mental. No informó de ello a la aerolínea a pesar de contar con las debidas instrucciones médicas, sino que lo oculto; esto puede deberse a que entre los pilotos existe un miedo respecto a cómo puede repercutir en su carrera el declarar tener problemas de salud o trastornos mentales que pudieran afectar su capacidad para desempeñar funciones de piloto ${ }^{5}$; como la toma de decisiones ante situaciones de estrés, buena comunicación en equipo de trabajo y estabilidad emocional para actuar de manera objetiva; tal fue el caso de la depresión grave que presentaba Lübitz, quien tenía antecedentes previos de depresión al punto de no haber aprobado el examen psicométrico para ingresar, y que hasta después de ser medicado con antidepresivos y antipsicóticos, logró aprobar, esto pudo generar en él un miedo de finalmente perder su licencia irrevocablemente.
Por consiguiente, la normativa en Europa ha adoptado medidas por medio de las cuales los pilotos de líneas aéreas deben tener un certificado médico (EASA Part-FCL Class 1), donde cada apartado debe ser cumplido para ser aprobado por la Comisión Europea ${ }^{6}$.

Andreas Lübitz ocultó que estaba de baja el día del accidente. Debido a esto, las normas que ha implementado la EASA para la validación y suspensión de certificados, incluyendo apartados como el informe breve e inmediato a la organización en la que opera el piloto en caso de una limitación o suspensión de la licencia, además de ciertas condiciones por escrito, donde notifique el motivo para el cese de operaciones y determinando bajo qué condiciones la suspensión será levantada, y que, hasta que no se demuestre que la causa de la suspensión ha sido rectificada, con una evaluación especial aprobada por la autoridad competente, según sea la severidad del problema, no se le permitirá restablecer sus operaciones como piloto. En caso de no haber ninguna otra limitante, la autoridad responsable informaría del cese de la suspensión?.

Entre los síntomas presentados en la depresión en los hombres, que difieren a los presentados en las mujeres, se encuentra la inhibición en el trabajo, fatiga y problemas de salud, retraso en la activación psicomotora, menosprecio de su capacidad personal, siendo estos los principales síntomas ${ }^{8}$.

La depresión masculina es una de las principales causas de suicidio en el mundo, debido al mal manejo de los medicamentos prescritos, al volverse una depresión crónica o severa como la presentada en Lübitz; el médico al no tomar este tipo de patología como un amenaza para el paciente, estaría atentando contra la sociedad de igual manera por las conductas y síntomas presentados en el paciente, esto se ve asociado a la alta comorbilidad con consumo prolongado de sustancias que a su vez incrementa el deterioro de los síntomas depresivos.

Una vez detectada la enfermedad en el sujeto la prevención secundaria puede ser aplicada para así evitar el progreso de esta misma y prevenir futuras complicaciones. Entre éstas se incluyen tratamientos psicológicos, como la activación conductual, la terapia cognitiva conductual y la psicoterapia interpersonal o antidepresivos; como los inhibidores selectivos de la recaptación de serotonina y los antidepresivos tricíclicos, todo esto recomendado por la Organización Mundial de la Salud ${ }^{9}$. 
En el caso presentado en el documental, durante las investigaciones realizadas hacia el copiloto, Lübitz, quien ya había sido diagnosticado con depresión severa, y era tratado con fármacos y terapias psicológicas, sin embargo 7 meses antes del accidente fue aprobado por los médicos de la aerolínea para poder volar. Igualmente, durante la investigación se encontró que durante los últimos meses había acudido a 41 doctores aquejándose de síntomas como pérdida de visión, probablemente a consecuencia de una psicosis previamente diagnosticada, gracias a este diagnóstico se infiere que presentaba una distorsión de la realidad y un grado de hipocondría.

Según los informes oficiales, Lübitz se encontraba bajo tratamiento con antideprecivos presuntamente mirtazapina a dosis máxima ya que referia insomino, ansiedad, y miedo inminente por la perdida de su visión la cual todos los médicos descartaron como entidad orgánica ${ }^{10}$.

Desde el nivel terciario, los eventos que se pudieron haber prevenido son: el no haber sido la aerolínea informada acerca de la condición del copiloto.

Entre las maneras de limitar el daño se encuentran internar al copiloto al psiquiátrico, el asegurarle a los pilotos que no perderán licencia o la posición laboral por llevar un tratamiento psicológico, donde al finalizar su tratamiento sean reintegrados y puedan presentar sus inquietudes a la aerolínea; aunado a la elaboración de protocolos que abarquen medidas de seguridad las cuales garanticen el bienestar físico de la tripulación y de los pasajeros, educación psicológica para las azafatas de grado profesional, exámenes más frecuentes (dos veces por mes) para poder limitar el daño hacia terceros, como en caso de Lübitz donde cometió homicidio de 149 personas y se quitó la vida.

El piloto refería la percepción de zonas oscuras o manchas en su campo visual, lo cual pudiera ser compatible con la presencia de escotomas o de simples manchas ciegas. En su correspondencia con su médico, Lubitz expresó su temor a estar perdiendo la visión, en un tono severo que reflejaba pensamientos obsesivos al respecto.

Los escotomas son, de acuerdo con la organización panamericana de la salud, defectos localizados comunes en las enfermedades retinianas y el glaucoma, así como en las enfermedades del nervio óptico, en otras ${ }^{11}$.

Según los estudios realizados por Joseph Allen 5 , en los que evalúa de manera anónima a 3,500 pilotos de diferentes nacionalidades, arrojaron resultados preocupantes, donde de los 1,848 que completaron las preguntas sobre salud mental; 233 (12.6\%) cumplen los criterios de probable depresión, 75 (4.1\%) reportaron tener pensamientos suicidas en las dos semanas previas; además, de 1,430 quienes reportaron haber trabajado en una aerolínea en los pasados 7 días antes de la encuesta, 193 (13.5\%) cumplían los criterios de posible grado de depresión.

Sin embargo, después de haber sido valorado por diversos especialistas, Lubitz había sido tratado de manera conservadora, como sucede en los casos en los que existen manchas ciegas, las cuales se consideran autolimitadas y de resolución espontánea.

Es pertinente mencionar que el antecedente de tratamiento con dosis máximas de Mirtazapina, pudiera asociarse a la presencia de escotomas, pero únicamente en los casos en los cuales el paciente curse con glaucoma, lo cual no correspondería con el estado de Lubitz.

Las tendencias actuales en medicina preventiva proponen las implementar y promocionar las medidas de lifestyle medicine las cuales se enfocan principalmente en promover la salud física, prevenir enfermedades con cambios en el comportamiento y actitudes relacionadas con la salud mental12.

La promoción de la salud mental puede partir desde la mejora en la actividad física y cambios en la dieta y se ha demostrado que estos mismos son efectivos en en el tratamiento de problemas de salud mental $y$, a veces, se prefiere por su falta de estigma asociado y menos efectos secundarios y complicaciones en comparación con el tratamiento psicológico o farmacológico ${ }^{12}$.

La campaña Act-Belong-Commit se propone como una promoción a la salud en la comunidad. Parte de la teoría de que nos volvemos personas con salud mental, si realizamos actividades que promuevan la salud metal. El "actuar" propone mantenerse alerta y comprometido mental, social, espiritualmente y físicamente activo. "Pertenecer" busca que la persona desarrolle un fuerte sentido de pertenencia al procurar amistades, integración a grupos y participar en actividades comunitarias. "Compromiso" es que las personas busquen cosas que le den sentido y propósito en la vida, como aceptar nuevos retos, ponerse nuevas metas, apoyar causas y ayudar a otros ${ }^{12}$.

\section{Discusión}

Hemos analizado las aportaciones que la medicina preventiva puede ofrecer en la prevención del suicidio a propósito del caso presentado en Mayday: catástrofes 
aéreas, en el cual se responsabilizó al copiloto de impactar intencionadamente el avión. El copiloto había sido diagnosticado con depresión mayor y siendo tratado con dosis altas de fármacos antidepresivos con efecto para regular el insomnio; adicionalmente, el copiloto abrigaba pensamientos obsesivos en referencia a su percibida afectación visual que aparentemente era de naturaleza psicosomática.

El estilo de vida del personal aéreo de las compañías comerciales implica un continuo cambio de actividades, dificulta el establecimiento de rutinas fijas tanto para ejercer actividades rutinarias individuales como para la participación social. En este sentido y considerando la responsabilidad que recae en los pilotos acerca de la vida y el bienestar de otras personas, el estilo de vida de los pilotos aéreos es comparable con la del personal de las fuerzas armadas o el personal médico en formación. La medicina preventiva ha considerado que las personas pertenecientes a estos gremios de profesionales se encuentran en un mayor riesgo tanto de depresión como de ansiedad y es por ello que se han generado escalas diagnósticas y recomendaciones preventivas que incluyen las estrategias del llamado "lifestyle medicine"13.

El lifestyle medicine se define como la aplicación de los principios ambientales, conductuales, médicos y de motivación para el manejo de problemas de salud relacionados con el estilo de vida en un entorno clínico e implica diversas estrategias de reducción del estrés, recreación y pertenencia a grupos sociales. La pertenencia cobra una gran importancia en la vida de los pilotos, debido a que el continuo cambio de sedes vinculado a la alteración de los ciclos de vigilia y sueño dificultan que ellos sean capaces de sentirse en un ambiente de hogar y exacerban los trastornos del sueño ${ }^{14}$.

La pertenencia a un grupo social, el ser como individuo y el ser en comunidad, de acuerdo con la filosofía personalista de Mounier es una cualidad de la persona humana como un ser complejo. Posiblemente, la afectación de dicha cualidad pudo incidir de manera negativa sobre la salud mental del joven copiloto y quizás incluso, haber influido sobre la decisión premeditada de no solo acabar con su propia vida, sino de terminar con la de las personas que iban a bordo del avión ${ }^{15}$.

Si bien los eventos de homicidios-suicidios, como el que en el presente artículo se presenta, se consideran hechos extremadamente raros, su impacto justifica que sean analizados a profundidad y que se tomen medidas para su prevención. Cinco de los seis eventos de homicidios y suicidios de una muestra de pilotos estudiada por
Kennedy y colaboradores sucedieron después de que los responsables se quedaran solos en la cabina y entre los factores asociados se identificaron tanto los conflictos legales como financieros, el estrés ocupacional, las enfermedades mentales y la disfunción de pareja. En el caso del copiloto de Germanwings, todos esos factores, excepto la disfunción de pareja, estuvieron presentes ${ }^{16}$.

Las condiciones emocionales y el estado de salud mental, así como las restricciones para pilotar asociadas a los fármacos que se habían indicado al copiloto debieron haber sido comunicadas oportunamente por parte de los médicos que trataron a Lubitz, sin embargo, en un intento por proteger la privacidad del paciente, no existieron medidas por parte de los profesionales de la salud por las cuales se aseguraran de que la compañía se había enterado de las condiciones de salud del copiloto. Las guías de práctica clínica para la prevención de los suicidios, son contundentes al mencionar que en caso de que exista riesgo de suicidio u homicidio, está bien justificado romper el secreto profesional17.

A partir del caso aquí presentado, la industria de la aviación puso en vigor las medidas preventivas que surgieron de las recomendaciones emitidas por los expertos que analizaron la trágica historia del germanwings, entre ellas, las medidas para facilitar la comunicación entre los evaluadores de la salud del personal con las compañías aéreas y los protocolos de conducta en el interior de la cabina de los aviones.

En un estudio transversal anonimizado, los autores reportaron que $4,1 \%$ de los pilotos informaron tener pensamientos suicidas, mientras un número significativo de pilotos activos padecían de síntomas depresivos. En dicha investigación, los autores recomendaron que las organizaciones aéreas aumentasen su apoyo para el tratamiento preventivo de salud mental y facilitasen que sus pilotos adoptasen medidas de higiene del sueño para reducir las alteraciones del ritmo circadiano ${ }^{5}$.

\section{Conclusión}

En conclusión, tomar las medidas preventivas necesarias para combatir la depresión, desde los distintos niveles, considerando como primaria la educación acerca de este trastorno para la concientización y erradicación de la depresión y conductas suicidas, medida secundaria la detección a tiempo de los primeros síntomas para evitar su evolución, y con esto la y rehabilitación y reintegración a la sociedad, evitando así un daño a la comunidad. 


\section{Referencias}

1. Botto A, Acuña J, Jiménez JP. La depresión como un diagnóstico complejo: Implicancias para el desarrollo de recomendaciones clínicas. Rev Méd Chile. 2014, 142(10): 1297-305.

2. Instituto Nacional de la Salud Mental de los Estados Unidos. Depresión. 2009; 09 (3561): p. 2-6

3. Belmaker RH, Agam G. Major Depressive Disorder. N Engl J Med. 2008; 358 (1): 55-68.

4. Bousoño $M$, Baca $E$, Álvarez $E$, Eguiluz I, Martín $M$, Roca $M$, et al. Complicaciones de la depresión a largo plazo. Actas Esp Psiquiatr. 2008; 36 (Suppl. 2):44-52.

5. Wu AC, Donnelly-McLay D, Weisskopf MG, McNeely E, Betancourt TS, Allen JG. Airplane pilot mental health and suicidal thoughts: a cross-sectional descriptive study via anonymous web-based survey. Environ Health. 2016;15(1):121.

6. Así es el test psicológico de un piloto: “¿Tiene usted impulsos homicidas?" Ecodiario, el Economista [Internet]. 27 de marzo de 2015 [citado el 13 e3 agosto de 2018].

7. FSTD qualification certificate: suspension and limitation. En European Aviation Safety Agency, editor. Acceptable Means of Compliance (AMC)and Guidance Material (GM) Part-ARA; 2016.

8. Londoño C, Peñate W, González M. Síntomas de depresión en hombres. Universitas Psychologica [Internet]. 2017, 16(4)

9. Centro de prensa/Notas descriptivas. Organización Mundial de la Salud. [Internet]. Depresión. 22 de marzo de 2018. [citado el 13 de agosto de 2018] 10. Müller E. (2016) La fiscalía francesa detalla los problemas mentales del piloto Lubitz: El alemán, que estrelló un avión de la compañía Germanwings con 149 personas a bordo, casi no dormía. El país 7 de marzo de 2018. [citado el 24 de septiembre de 2018].

11. Berling I, Isbister GK. Mirtazapine overdose is unlikely to cause major toxicity. Clin Toxicol (Phila). 2014;52(1):20-4

12. Donovan RJ, Anwar-McHenry J. Act-Belong-Commit: Lifestyle Medicine for Keeping Mentally Healthy. Am J Lifestyle Med. 2014;10(3):193-99.

13. Mechanick, J. I., \& Kushner, R. F. (2016). Why lifestyle medicine? En Mechanick JL, Kushner RF, editors. Lifestyle Medicine: A Manual for Clinical Practice. New York: Springer; 2016. p. 1-8.

14. Rippe JM. Lifestyle medicine. 1st ed. Malden, Ma: Blackwell Science; 1999.

15. Sgreccia, E. Persona humana y personalismo. Cuad Bioet. 2013; 24(1):115-23. 16. Kenedi C, Friedman SH, Watson D, Preitner C. Suicide and murder-suicide involving aircraft. Aerosp Med Hum Perform. 2016; 87(4):388-96.

17. National Institute for Health and Clinical Excellence. (2009). Depression in Adults: Recognition and Management. Clinical guideline [CG90]. Published date: October 2009 Updated: April 2018

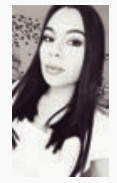

María Jesús Aldecua Tejero es estudiante de medicina en la Universidad Marista de Mérida, México. Actualmente cursa el quinto semestre de la carrera de medicina y está participando en rotaciones en diferentes áreas clínicas en unidades médicas familiares del Instituto Mexicano del Seguro Social.

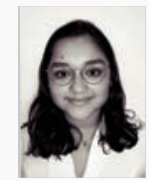

Citlalli Abigail García Mezo es estudiante de medicina de la Universidad Marista de Mérida, México, cursa el quinto semestre de la carrera Medicina, y actualmente se encuentra rotando en hospitales de primer nivel del Instituto Mexicano del Seguro Social.

Martín de Jesús Inurreta Díaz es médico cirujano, actualmente realiza su servicio social en investigación el cual obtuvo bajo concurso nacional de la Comisión Interinstitucionalista para la Formación de Recursos Humanos para la Salud. Ha tenido de trabajar en diferentes proyectos de investigación los cuales ha presentado en foros médicos especializados nacionales e internacionales.

Leticia Lizama Rubio, estudiante de la Universidad Marista de Mérida, Yucatán, México. Actualmente se encuentra cursando el tercer año de la Licenciatura de Medicina, iniciando prácticas rotando en los diversos servicios de unidades de Medicina Familiar (IMSS), ha obtenido certificaciones en medicina preventiva de la organización panamericana de la salud y buenas practicas clínicas (NIDA Clinical Trials Network).

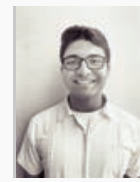

Carlos Daniel Velasco Bonilla es estudiante de medicina en la Universidad Marista de Mérida, México, cursa el tercer año de la carrera de Medicina, se encuentra rotando por servicios diversos de unidades de Medicina Familiar del Seguro Social Mexicano. 